\title{
Nanostructured transition metal nitride composites as energy storage material
}

\author{
YUE YanHua ${ }^{\dagger}$, HAN PengXian ${ }^{\dagger}$, DONG ShanMu, ZHANG KeJun, ZHANG ChuanJian, \\ SHANG ChaoQun \& CUI GuangLei*
}

Qingdao Institute of Bioenergy and Bioprocess Technology, Chinese Academy of Sciences, Qingdao 266101, China

Received February 7, 2012; accepted April 1, 2012; published online July 2, 2012

\begin{abstract}
There are growing demands for the next generation lithium ion batteries with high energy density as well as high power performance for renewable energy storage and electric vehicles application. Recently, nanoscale materials with outstanding energy storage capability have received considerable attention due to their unique effect caused by the reduced dimensions. This review describes some recent developments of our group in research of transition metal nitride nanocomposites in application of energy storage, especially for lithium ion battery and supercapacitor. The strategies of mixed conduction (electron and ion) network with a favorable charge transportation interface in the design of the nanocomposites for such devices are highlighted.
\end{abstract}

nanocomposites, transition metal nitride, lithium ion battery, super capacitor

Citation: Yue Y H, Han P X, Dong S M, et al. Nanostructured transition metal nitride composites as energy storage material. Chin Sci Bull, 2012, 57: 4111-4118, doi: $10.1007 / \mathrm{s} 11434-012-5301-1$

Our present energy policy still mainly based on burning fossil fuels, inevitably poses a serious concern due to $\mathrm{CO}_{2}$ related global warming. Accordingly, efforts aimed at ensuring efficient use of renewable energy sources and replacement of internal combustion engines with electric motors for the development of sustainable vehicles, such as hybrid electric vehicles (HEVs), plug in hybrid electric vehicles (PHEVs), and ultimately full electric vehicles (EVs), are in progress worldwide [1]. Exploration of alternative, green energy sources, such as solar, wind and geothermal, regains the side support of energy storage system that can compensate their intermittent characteristics. It is now generally accepted that among the various choices, the most suitable are lithium ion batteries (LIB). LIB is portable devices capable of delivering the stored chemical energy as electrical energy with high conversion efficiency and without any gaseous emission. Moreover, LIB offers the most promising option to power efficiently HEVs and EVs. In

$\dagger$ These authors contributed equally to this work.

*Corresponding author (email: cuigl@qibebt.ac.cn) this scenario, LIB and electrochemical supercapacitor are among the most promising energy storage devices that can meet the demands of high power, high energy and long cycle life.

Transition metal nitride nanocomposites are considered to be the promising anode material in LIB for their low lithium intercalation potential, high reversible reaction and high capacity. $\mathrm{Li}_{x} \mathrm{M}_{y} \mathrm{~N}(\mathrm{M}=\mathrm{Fe}, \mathrm{Co}, \mathrm{Ni}, \mathrm{Cu}, \mathrm{Mn})$ exhibits high ion conductivity and variable metal valence. Among them, $\mathrm{Li}_{3-x} \mathrm{Cu}_{x} \mathrm{~N}$ and $\mathrm{Li}_{3-x} \mathrm{Co}_{x} \mathrm{~N}$ give a reversible capacity of 650 and $560 \mathrm{mAh} \mathrm{g}^{-1}$, respectively. However, their power density and cyclic performance suffer from the volume change during charge/discharge process, leading to poor cycle performance. Fu et al. in Fudan University did a good job in a series of transition metal nitrides such as $\mathrm{Fe}_{3} \mathrm{~N}$ [2], $\mathrm{Ni}_{3} \mathrm{~N}$ [3], $\mathrm{CrN}$ [4], VN [5] and their composites [6] indicating that transition metal nitrides with higher valence give a higher capacity. The capacity of $\mathrm{CrN}$ and $\mathrm{VN}$ could be 1500 mAh g ${ }^{-1}$, much higher than that of $\mathrm{Fe}_{3} \mathrm{~N}$ and $\mathrm{Co}_{3} \mathrm{~N}$ (400 $\left.\mathrm{mAh} \mathrm{g}^{-1}\right)$. However, the poor ion and electrical conductivities of $\mathrm{CrN}$ give rise to the high degree of polarization, 
which greatly limits its practical application [7]. To overcome these disadvantages and further enhance the energy density, the obtained electrode material must be of high specific capacity and low lithium intercalation/deintercalation potential [8]. So it is very interesting to investigate the energy storage capacity of VN given the above the excellent performance. Moreover, TiN represents a good conductivity and is favorable to enhance the rate performance of LIB, such as $\mathrm{Li}_{4} \mathrm{Ti}_{5} \mathrm{O}_{12} / \mathrm{TiN}$ [9] and Si/TiN [10]. Recently, TiNgraphene hybrid has also been studied for its electric catalysis in dye-sensitized solar cell and biosensor [11].

Herein, the review describes some recent developments of our group in research of transition metal nitride nanocomposites in application of energy storage, especially for LIB and supercapacitor. The strategy of mixed conduction (electron and ion) with a favorable charge transportation interface in the design of the nanocomposites for such devices is highlighted.

\section{Transition metal nitrides as anode material in LIB}

Generally, there are three dynamic processes for $\mathrm{Li}^{+}$intercalation in LIB: (1) $\mathrm{Li}^{+}$motion through the electrolyte; (2) migration in the electrolyte/electrode interface; and (3) chemical diffusion in the bulk of electrode material, as shown in Figure 1. Among the three processes, (3) is the decisive one for the whole $\mathrm{Li}^{+}$transportation and is defined as the function: $\tau=L^{2} / 2 D$, where $\tau$ is the diffusion time, $L$ is the length of diffusion path and $D$ is diffusion coefficient of the electrode material. Once the size of the material is shortened, the transport length and the chemical diffusion resistance are decreased. Consequently, superior electrochemical performance is obtained [12-14].

Nano-sized materials were demonstrated to be of low electrochemical polarization, high reversible capacity, and negligible volume change effect during the charge/discharge process [15-17]. As an excellent electrode material, it requires fast lithium ion transportation and mixed electrical conductivity $[18,19]$. Therefore, a series of nanostructrued composite materials are designed, in which ion and electron can be transported efficiently. Thus high energy density and high power density are obtained simultaneously (Figure 2).

A series of transition metal nitrides, such as GeN [20], $\mathrm{Zn}_{3} \mathrm{~N}_{2}$ [21] and $\mathrm{Cu}_{3} \mathrm{~N}$ [22], have been studied, the lithium storage mechanism of these composites is described as

$$
\begin{gathered}
\mathrm{M}_{x} \mathrm{~N}_{y}+3 y \mathrm{Li}^{+}+3 y \mathrm{e}^{-} \rightarrow x \mathrm{M}+y \mathrm{Li}_{3} \mathrm{~N}, \\
\mathrm{M}+z \mathrm{Li}^{+}+z \mathrm{e}^{-} \rightarrow \mathrm{Li}_{z} \mathrm{M}
\end{gathered}
$$

Some of the transition metal nitrides have a low electrical conductivity and volume change effect during charge/discharge process, which result in their low power density and poor cyclic performance. It is known that TiN shows good conductivity and high temperature stability [23]. Meanwhile, $\mathrm{VN}$ is reported to display considerable capacity. Based on this, Joachim Maier (Max-Planck-Institute for Solid State Research) and our team cooperated to explore in the preparation of Ti-V-N/C where the carbon is coated on the surface (Figure 3) to inhibit the volume change. The Ti-V-N/C composite with a particle size of $8 \mathrm{~nm}$ exhibits good rate performance: 639 and $95 \mathrm{mAh} \mathrm{g}^{-1}$ at the rates of $74.4 \mathrm{~mA} \mathrm{~g}^{-1}$ and $22.32 \mathrm{~A} \mathrm{~g}^{-1}$, respectively.
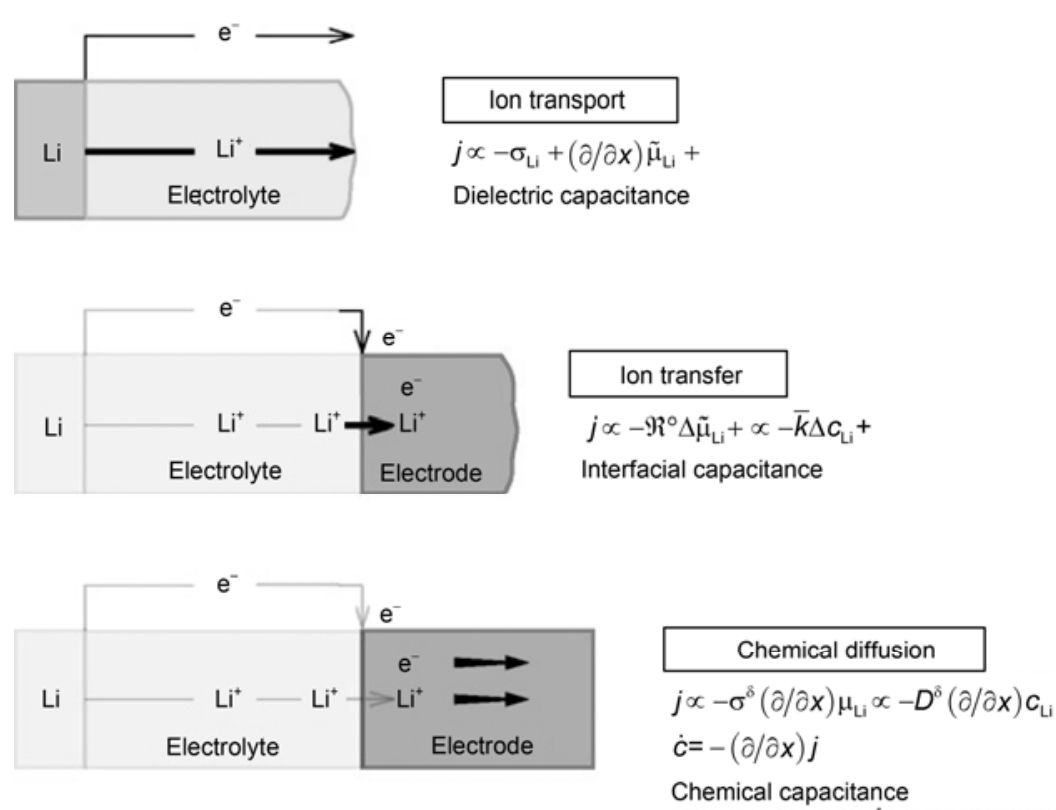

Figure 1 Three dynamic processes for $\mathrm{Li}^{+}$in LIB. Reprinted from [12], Copyright 2007, with permission from Elsevier. 


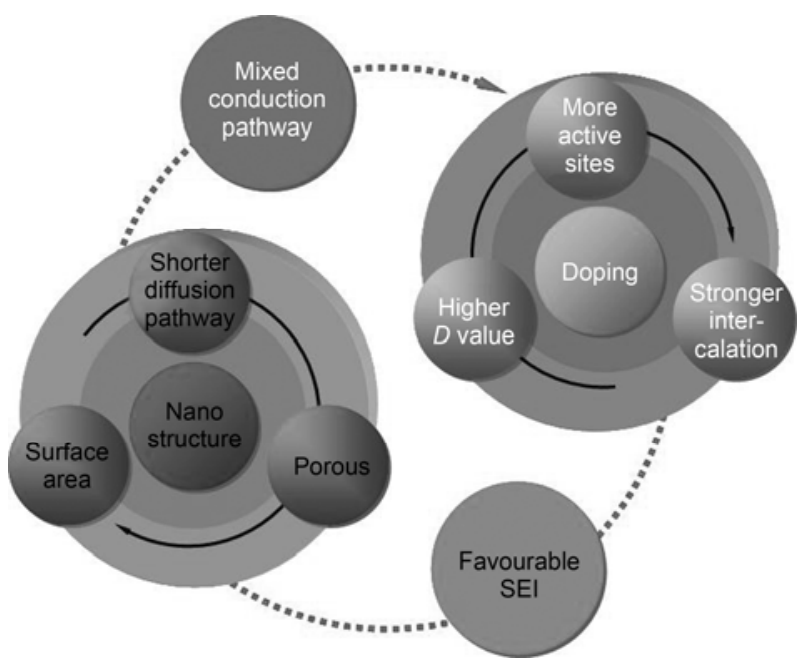

Figure 2 Schematic map of the design for hybrid transfer material.

Graphene (G), a two-dimensional nanostructure of carbon, has attracted a great deal of attention since it was experimentally discovered in 2004 . G possesses a high surface area to volume ratio, extraordinary electronic transport properties, flexibility and chemical stability, and is very promising in applications such as solar cells, sensors, batteries and supercapacitors [25]. Our team has done much work on exploring structure-performance effect of nitrogen-doped G (Figure 4), which indicates that the introduction of nitrogen atoms highly improves the lithium intercalation capability of $\mathrm{G}$ [26]. The reason is that the structural defects caused by $\mathrm{N}$ atom can accommodate much more $\mathrm{Li}$ ion and the decreased active sites on the $\mathrm{G}$ edge lead to reduced irreversible capacity. It was reported by Ogumi [27] that the interface resistance plays an important role in the course of lithium intercalation. In our case, N-doping in $\mathrm{G}$ greatly reduces the interface, and thus facilitates the electrochemical dynamic process.

Subsequently, graphene G/TiN hybrid is obtained through in situ synthesis. G/TiN hybrid anode delivers a reversible capacity as high as $646 \mathrm{mAh} \mathrm{g}^{-1}$ at $20 \mathrm{~mA} \mathrm{~g}^{-1}$ and exhibits an enhanced initial coulomb efficiency of $75 \%$, much higher than that of pure graphene $(\mathrm{G}, 52 \%)$ in the first cycle (Figure 5(a)). At a current density of $2000 \mathrm{~mA} \mathrm{~g}^{-1}$, the hybrid anode still retains $325 \mathrm{mAh} \mathrm{g}^{-1}$ while that of $\mathrm{G}$ is only $98 \mathrm{mAh} \mathrm{g}^{-1}$ (Figure 5(b)) [28]. It is demonstrated that the G/TiN hybrids display superior electrochemical performance owing to the highly efficient mixed (electron and $\mathrm{Li}^{+}$) conducting network. The internal defects between $\mathrm{G}$ layers induced by nitrogen-doping in G/TiN may improve reversible Li storage, whereas the catalytic sites on the surface of $\mathrm{G}$ related to the decomposition of electrolyte may be occupied by TiN, leading to decreased irreversible capacity. AC impedance also confirmed that both the solid electrolyte interface and charge transfer resistance are decreased after the anchoring of TiN nanoparticles [29,30]. In addition, the conductivity of G/TiN and graphene is obtained by fourprobe method as 2.36 and $0.25 \mathrm{~S} \mathrm{~cm}^{-1}$, respectively.

Accordingly, N-dopped G/VN (VN-G) hybrid is also studied [31]. It is very interesting that when the current density returns to $42 \mathrm{~mA} \mathrm{~g}^{-1}$ after the rate test, the reversible capacity of all the samples is demonstrated to show a significant increase than the original value (Figure 6(a)). In order to clarify the reason for the result, XRD is applied after cycle measurement as shown in Figure 6(b). The XRD analyses reveal a shift of electrochemically reformed "VN" peaks towards larger angles (with respect to the initial VN phase), corresponding to a lattice contraction after cycling. It may be attributed to the formation of $\mathrm{L} \mathrm{Li}_{x} \mathrm{~V}_{1-x} \mathrm{~N}$ phase as the substitution of $\mathrm{Li}^{+}$ions, of about $0.68 \AA$ in diameter, for larger vanadium ions, resulting in a lattice contraction.

Figure 7(a) is a typical bright-field transmission electron micrograph, showing that the nanoparticles range from 15 to $100 \mathrm{~nm}$ in diameter. Electron energy-loss spectroscopy (EELS) analyses are conducted in two different regions, denoted as zone 1 and zone 2 in Figure 7(a), to detect the

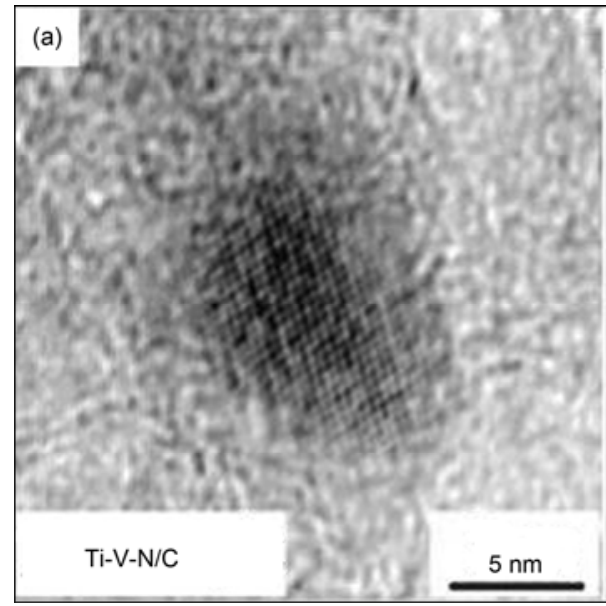

(b)

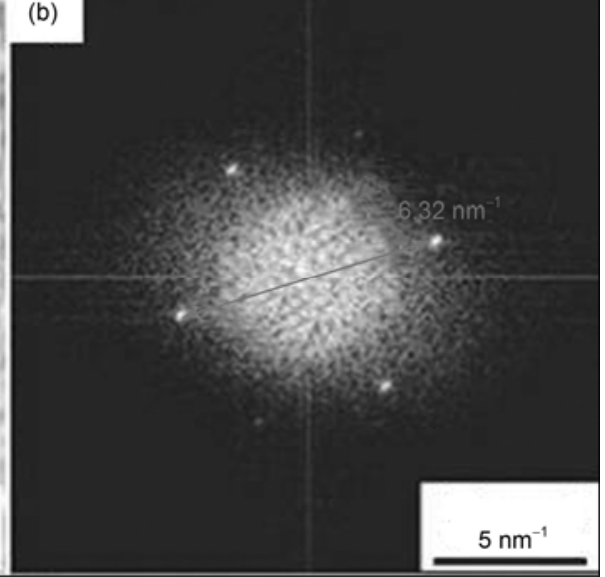

Figure 3 (a) High revolution TEM image; (b) electron diffraction pattern of Ti-V-N/C nanocomposite. Reprinted with permission from [24]. 


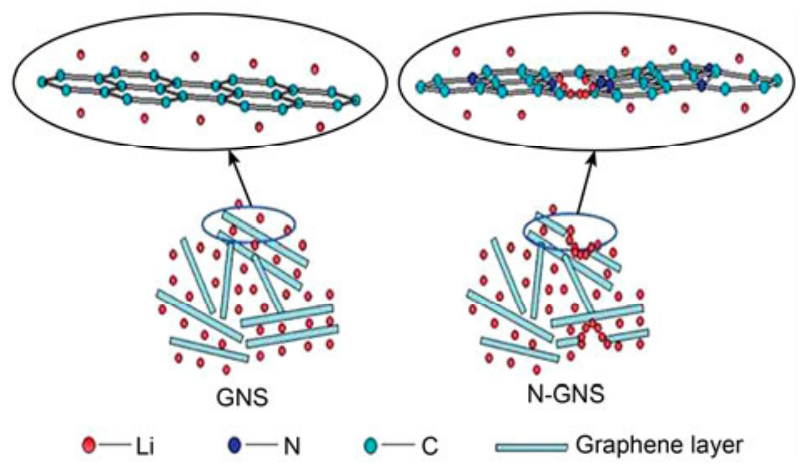

Figure 4 The mechanism of lithium storage in N-doped G. Reprinted with permission from [26].

chemical changes of nitride and vanadium after cycling, as shown in Figure 7(b) with a magnified version of N-K edge inset. Normalizing the two spectrum using N-K edges gives us a clear clue that the V-L edge is higher in zone 1 than that in zone 2, indicating that the content of vanadium is higher in zone 1. This leads us to a conclusion that more lithium is incorporated into the lattice by forming lithium nitrides in smaller and thinner nanoparticles (zone 1).

The nano-sized electrode material transforms some inactive materials into lithiation active ones, exhibiting higher capacity than commercialized graphite. However, the electrochemical dynamics of these materials still need to be enhanced. To solve this problem, we designed nanostructured composites with highly efficient mixed (electron and $\mathrm{Li}^{+}$) conducting network, optimized electrode interface and homogenous structure, which are of crucial importance for high energy density, high power density, long cycle life and other special needs.

\section{Transition metal nitrides as active material in supercapacitor}

As an excellent electrode material of supercapacitor, it must have high specific surface area and good conductivity. In the past few decades, many efforts had been made in the research of mesoporous materials with proper surface area
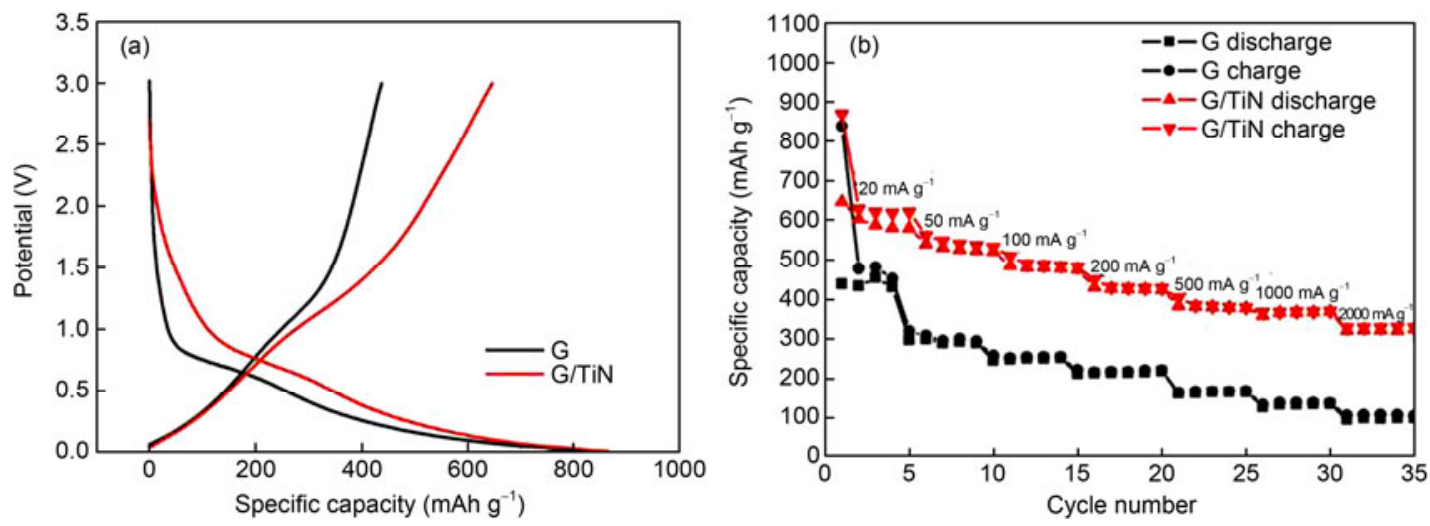

Figure 5 (a) First charge/discharge profiles of G/TiN and G at the current density of $20 \mathrm{~mA} \mathrm{~g}^{-1}$; (b) rate performance of G/TiN and G. Reprinted with permission from [28].
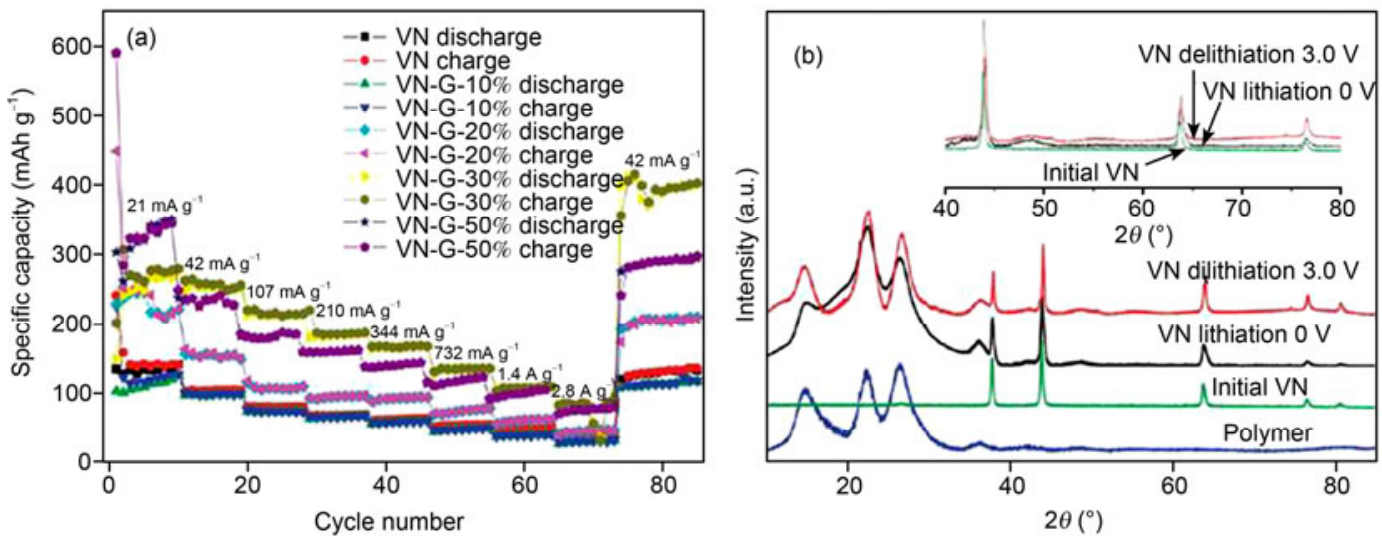

Figure 6 (a) Cycling and rate performance of VN-G hybrid materials and pristine VN electrodes cycled in EC/DMC solution containing 1 mol/L LiPF6; (b) $\mathrm{XRD}$ patterns of the $\mathrm{VN}$ electrode obtained at the end of lithiation at $0 \mathrm{~V}$ and at the end of delithiation at $3.0 \mathrm{~V}$ compared to the initial $\mathrm{VN}$ electrode. Reprinted with permission from [31]. 

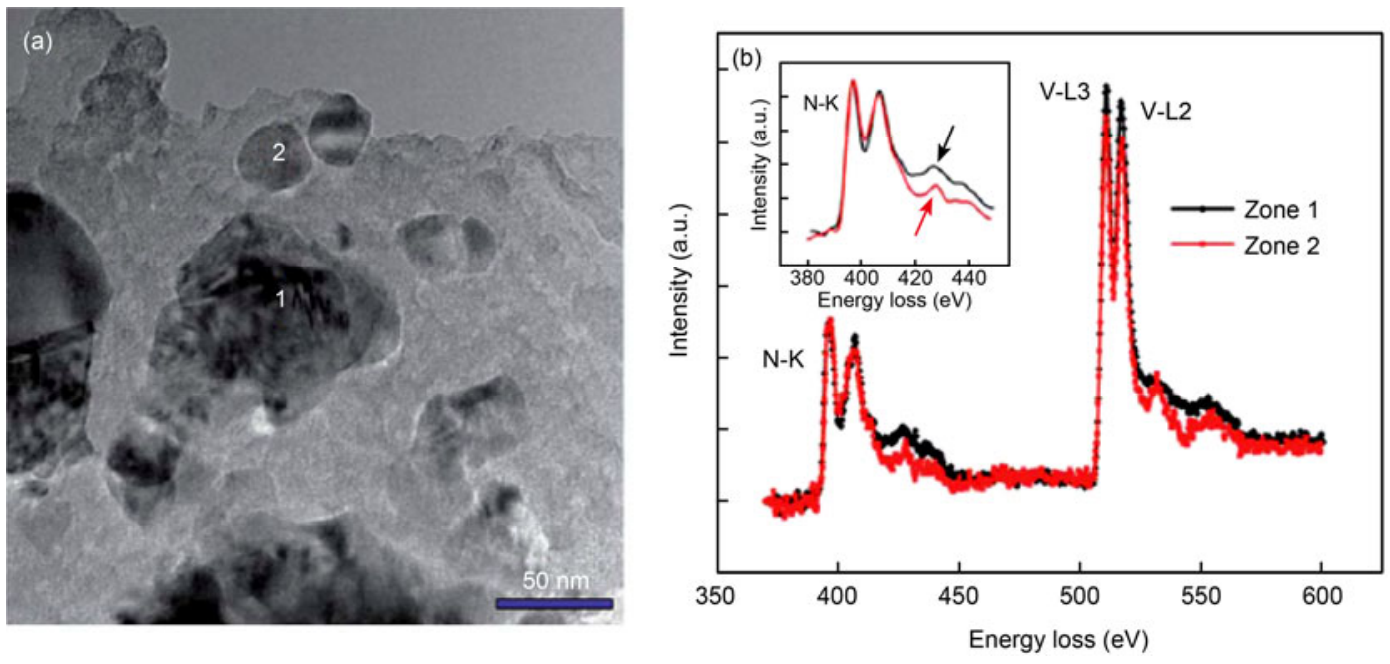

Figure 7 TEM and EELS analyses of the VN samples after cycles, indicating that lithium ions are incorporated into the vanadium nitride by extended cycling. Reprinted with permission from [31].

and accessible pores which will facilitate the mass transfer and ion conduction. Meanwhile, when high operation voltage is applied in nonaqueous supercapacitor, high energy density can be obtained [32]. With these in mind, mesoporous TiN nanoparticles and TiN-VN composites with different structures are prepared.

\subsection{Mesoporous TiN nanoparticles for supercapacitor}

TiN nanoparticles are often prepared from titanium oxide under ammonia atmosphere at high temperature. However, it is challenging to form a mesoporous structure, because the collapse of the nanopores during conversion and recrystallization severely hinders their formation. To address this issue, the general synthetic strategies mostly rely on the use of multiple templates. However, these approaches substantially require a tedious and time-consuming process of tem- plate formation and the necessity of template removal. We reported a facile route to directly convert $\mathrm{TiO}_{2}$ mesoporous spheres into TiN mesoporous spheres under ammonia atmosphere using cyanamide to retain the morphology (Figure 8) [33]. The single-electrode specific capacitance of the TiN are $112.3 \mathrm{~F} \mathrm{~g}^{-1}$ at the current densities of $0.1 \mathrm{~A} \mathrm{~g}^{-1}$. The energy density can reach $45.0 \mathrm{~W} \mathrm{~h} \mathrm{~kg}^{-1}$ at a power density of $150 \mathrm{~W} \mathrm{~kg}^{-1}$.

It can be expected that the mesoporous TiN spheres can serve as a promising electrode material for high energy supercapacitors. Furthermore, the mesoporous structure of TiN is potential framework for synthesizing composites with 3D mixed (electron and ion) conducting networks by introducing electro-active materials (e.g. $\mathrm{MnO}_{2}, \mathrm{RuO}_{2}, \mathrm{Si}$, $\mathrm{Sn}$ ) on its surface. Therefore, an excellent electrode material based on mixed conducting composites with a higher specific energy and power density can be expected, which is
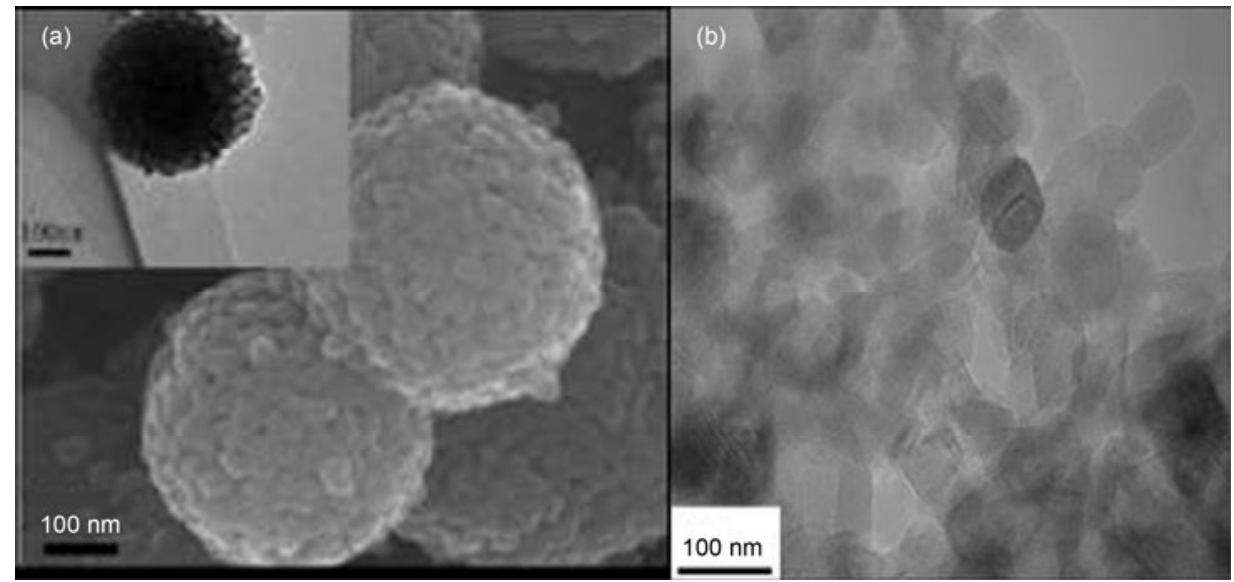

Figure 8 (a) SEM image and TEM image (inset) of the mesoporous TiN spheres with diameter range from 200 to $300 \mathrm{~nm}$; (b) HRTEM image of mesoporous TiN spheres with diameter range from 200 to $300 \mathrm{~nm}$. Reprinted with permission from [33]. 
highly desirable for LIB and supercapacitor applications.

\subsection{Mesoporous coaxial TiN-VN fibers of core-shell structures for supercapacitors}

As mentioned above, TiN possesses good conductivity while VN shows outstanding capacitance capability. Accordingly, TiN-VN fibers of core-shell structures were prepared by the coaxial electro-spinning, and subsequently annealed in the ammonia. TiN-VN fibers incorporated mesoporous structure into high electronic conducting transition nitride hybrids. The hybrids exhibit high specific capacitance $\left(2 \mathrm{mV} \mathrm{s}^{-1}\right.$, $\left.247.5 \mathrm{~F} \mathrm{~g}^{-1}\right)$ and good rate capability $\left(50 \mathrm{mV} \mathrm{s}^{-1}, 160.8 \mathrm{~F} \mathrm{~g}^{-1}\right)$ (Figure 9).

In addition, TiN/VN core-shell nanospheres are also prepared by a two-step strategy involving coating of commercial TiN nanoparticles with $\mathrm{V}_{2} \mathrm{O}_{5} \cdot n \mathrm{H}_{2} \mathrm{O}$ sols followed by ammonia reduction, as shown in Figure 10(a). The highest specific capacitance of $170 \mathrm{~F} \mathrm{~g}^{-1}$ is obtained when scanned at $2 \mathrm{mV} \mathrm{s}^{-1}$ and a promising rate capacity performance is maintained at higher voltage sweep rates (Figure 10(b)). The favorable specific capacitance may be attributed to the enhancement of electrical conductivity and nanostructured materials [35].

\subsection{One-dimensional $\mathrm{MnO}_{2} / \mathrm{TiN}$ nanotube coaxial arrays for supercapacitor}

Nowadays, innovative electrochemical energy storage electrodes for supercapacitors have been extensively studied. Among a variety of options, $\mathrm{MnO}_{2}$ is one of the most popular candidates, because of its high energy density, low cost, non-toxicity, and natural abundance. However, its applications in supercapacitors are hindered due to its poor rate capability arising from its low electronic conductivity. Therefore, a one-dimensional nanostructured coaxial composite of $\mathrm{MnO}_{2}$ and an electronic conductive material TiN is designed, based on the concept of designing an efficient, fast charge transportation network, and is expected to significantly enhance the rate performance of $\mathrm{MnO}_{2}$ electrodes. SEM images of TiN nanotubes (Figure 11(a),(b)) and the $\mathrm{MnO}_{2}$ / TiN coaxial nanostructure using a deposition potential of $0.7 \mathrm{~V}$ (Figure 11(c)) shows a uniform structure. The TiN nanotubes have outer diameters ranging from 65 to $75 \mathrm{~nm}$,

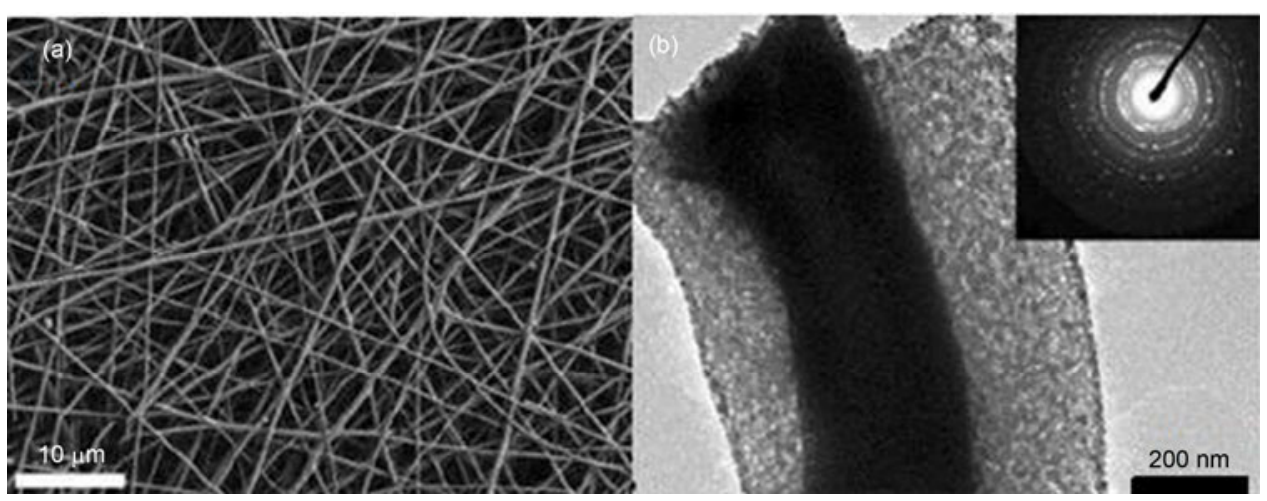

Figure 9 (a) SEM and (b) TEM images of mesoporous coaxial TiN-VN fibers of core-shell structure. Reprinted with permission from [34].
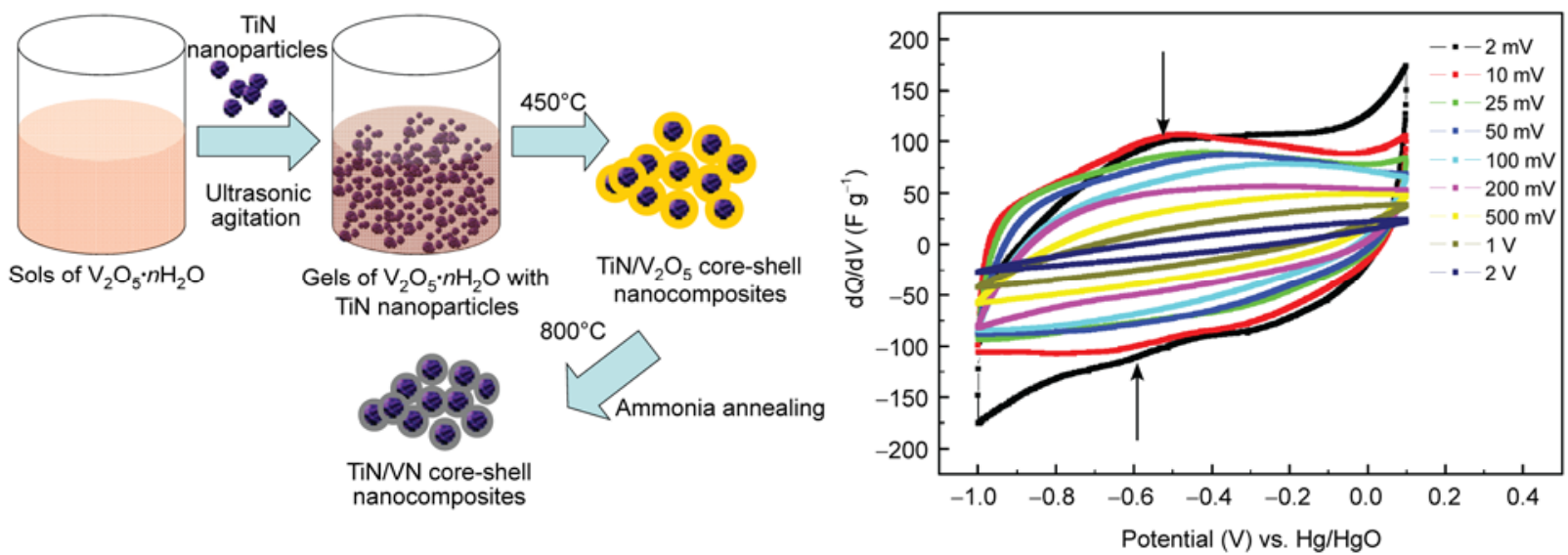

Figure 10 (a) Schematic illustration for the preparation of TiN/VN nanocomposite; (b) CV curves of TiN/VN composite in 1 mol/L KOH aqueous solution. Reprinted from [35]. Copyright 2011, with permission from Elsevier. 

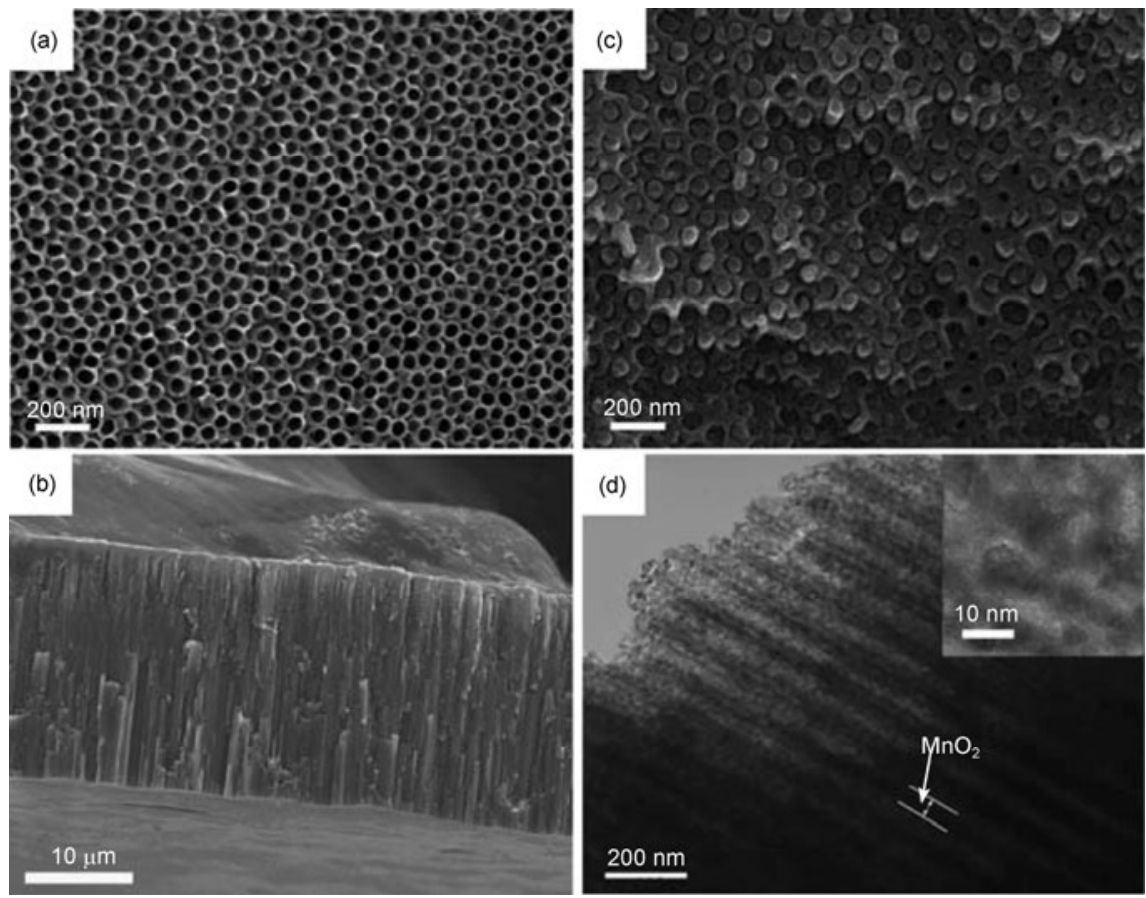

Figure 11 (a), (b) TiN nanotubes; (c), (d) coaxial $\mathrm{MnO}_{2} / \mathrm{TiN}$. Reprinted with permission from [36].

and the arrays are about $20 \mathrm{~mm}$ in length. $\mathrm{MnO}_{2}$ is evenly deposited into the nanotubes. The combination of mesoporous $\mathrm{MnO}_{2}$ and TiN in coaxial nanowire arrays has shown promising capacitance $\left(390.2 \mathrm{~F} \mathrm{~g} \mathrm{~g}^{-1}\right.$ at a current density of $400 \mathrm{~A} \mathrm{~g}^{-1}$ ), excellent rate capability (only $45 \%$ loss at a scan rate of $2000 \mathrm{mV} \mathrm{s}^{-1}$ compared with that at a scan rate of $2 \mathrm{mV} \mathrm{s}^{-1}$ ) [36].

\section{Conclusions}

High efficient electrode materials with nanostructure play an important role in the development of new energy storage devices. Building up materials for electrical energy storage is a huge challenge that has been taken on by our groups, starting from fundamental insight into structure and performance based on the concept of mixed conducting network. By constructing favorable electrode/electrolyte interface and assembling unique nanostructure, transition metal nitride nanocomposites exhibit excellent electrochemical performance. It is very meaningful and prosperous for the investigation of these materials in application of both LIB and supercapacitor.

This work was supported by the Hundred Talents Program of the Chinese Academy of Sciences, the National Basic Research Program of China (2011CB935703), Shandong Provincial Funds for Distinguished Young Scientist (JQ200906) and the National Natural Science Foundation of China (20971077).

1 Yan D Z. Effective way of new energy alternative to fossil fuels-
Low-carbon fuel emissions standards. City, 2010, 1: 74-75

2 Fu Z W, Wang Y, Yue X L, et al. Electrochemical reactions of lithium with transition metal nitride electrodes. J Phy Chem B, 2004, 108 : 2236-2244

3 Wang Y, Fu Z W, Yue X L, et al. Electrochemical reactivity mechanism of $\mathrm{Ni}_{3} \mathrm{~N}$ with lithium. J Electrochem Soc, 2004, 151: E162

4 Sun Q, Fu Z W. An anode material of $\mathrm{CrN}$ for lithium-ion batteries. Solid-State Lett, 2007, 10: A189-A193

5 Sun Q, Fu Z W. Vanadium nitride as a novel thin film anode material for rechargeable lithium batteries. Electrochim Acta, 2008, 54: 403-409

6 Sun Q, Fu Z W. $\mathrm{Cr}_{1-x} \mathrm{Fe}_{x} \mathrm{~N}(0 \leqslant x \leqslant 1)$ ternary transition metal nitrides as anode materials for lithium-ion batteries. Electrochem Solid-State Lett, 2008, 11: A233-A237

7 Sun Q. Studies on series of high valence transitional metal nitride as novel anode materials for lithium-ion battery. Master's Dissertation. Shanghai: Fudan University, 2009

8 Xin S, Guo Y G, Wan L J. Electrode materials for lithium secondary batteries with high energy densities. Sci Sin Chim, 2011, 41: 12291239

9 Snyder M Q, Trebukhova S A, Ravdel B, et al. Synthesis and characterization of atomic layer deposited titanium nitride thin films on lithium titanate spinel powder as a lithium ion battery anode. J Power Sources, 2007, 165: 379-385

10 Kim I, Kumta P N, Blomgren G E. Si/TiN nanocomposites: New anode materials for Li-ion batteries. Electrochem Solid-State Lett, 2000, 3: 493-496

11 Wen $\mathrm{Z}$, Cui S, Pu H, et al. Metal nitride/graphene nanohybrids: General synthesis and multifunctional titanium nitride/graphene electrocatalyst. Adv Mater, 2011, 23: 5445-5450

12 Maier J. Size effects on mass transport and storage in lithium batteries. J Power Sources, 2007, 174: 569-574

13 Aurbach D, Bruneel J L, Grondn J, et al. Common electro analytical behavior of $\mathrm{Li}$ intercalation processes into graphite and transition metal oxides. J Electrochem Soc, 1998, 145: 3024-3034

14 Balaya P. Size effects and nanostructured materials for energy applications. Energy Environ Sci, 2008, 1: 645-654

15 Linkov I, Steevens J. Nanomaterials: Risks and Benefits. Heidelberg: Springer, 2009

16 Li H, Wang Z X, Chen L Q, et al. Research on advanced materials for 
Li-ion batteries. Adv Mater, 2009, 21: 4593-4607

17 Guo Y G, Hu J S, Wan L J. Nanostructured materials for electrochemical energy conversion and storage devices. Adv Mater, 2008, 20: $2878-2887$

18 Gaberscek M J. Impact of electrochemical wiring topology on the kinetics of insertion electrodes. J Solid State Ionics, 2006, 177: 2647-2651

19 Guo Y G, Hu Y S, Maier J. Synthesis of hierarchically mesoporous anatase spheres and their application in lithium batteries. Chem Commun, 2006, 2783-2785

20 Pereira N, Balasubramanian M, Dupont L, et al. The electrochemistry of germanium nitride with lithium. J Electrochem Soc, 2003, 150: A1118-A1128

21 Pereira N, Klein L C, Amatucci G G. The electrochemistry of $\mathrm{Zn}_{3} \mathrm{~N}_{2}$ and LiZnN. J Electrochem Soc, 2002, 149: A262-A271

22 Pereira N, Dupont L, Tarascon J M, et al. Electrochemistry of $\mathrm{Cu}_{3} \mathrm{~N}$ with lithium. J Electrochem Soc, 2003, 150: A1273-A1280

23 Harrison J F. Electronic structure of the transition metal nitrides TiN, VN, and CrN. J Phys Chem, 1996, 100: 3513-3519

24 Cui G L, Gu L, Thomas A. A carbon/titanium vanadium nitride composite for lithium storage. ChemPhysChem, 2010, 11: 3219-3223

25 Geim A K, Novoselov K S. The rise of graphene. Nat Mater, 2007, 6 : 183-191

26 Wang H B, Zhang C J, Cui G L, et al. Nitrogen-doped graphene nanosheets with excellent lithium storage properties. J Mater Chem, 2011, 21: 5430-5434

27 Ogumi Z. A.c. impedance analysis of electrochemical lithium intercalation into highly oriented pyrolytic graphite. J Power Sources,
1997, 68: 227-231

28 Yue Y H, Han P X, Cui G L, et al. In situ synthesis of graphene/titanium nitride hybrid material with highly improved performance for lithium storage. J Mater Chem, 2012, 22: 4938-4943

29 Zhuang Q C, Chen Z F, Dong Q F, et al. Electrochemical impedance spectroscopy study of the first cathodic polarization process in graphite anode. Chin Sci Bull, 2006, 51: 17-20

30 Tian L L, Zhuang Q C, Li J, et al. Insertion and deinsertion of lithiumion extrusion in graphene. Chin Sci Bull, 2011, 56: 1431-1439

31 Zhang K J, Wang H B, Cui G L, et al. A hybrid material of vanadium nitride and nitrogen-doped graphene for lithium storage. J Mater Chem, 2011, 21: 11916-11922

32 Jang B Z, Liu C G, David N, et al. Graphene surface-enabled lithium ion-exchanging cells: Next-generation high-power energy storage devices. Nano Lett, 2011, 11: 3785-3791

33 Dong S M, Chen X, Cui G L, et al. Facile preparation of mesoporous titanium nitride microsphres for electrochemical energy storage. ACS Appl Mater Interface, 2011, 3: 93-98

34 Zhou X H, Shang C Q, Cui G L, et al. Mesoporous coaxial titanium nitride-vanadium nitride fibers of core-shell structures for high-performance supercapacitors. ACS Appl Mater Interface, 2011, 3: 30583063

35 Dong S M, Chen X, Cui G L, et al. TiN/VN composites with core/ shell structure for supercapacitors. Mater Res Bull, 2011, 46: 835839

36 Dong $\mathrm{S} \mathrm{M}$, Chen X, Cui G L, et al. One dimensional $\mathrm{MnO}_{2} /$ titanium nitride nanotube coaxial arrays for high performance electrochemical capacitive energy storage. Energy Environ Sci, 2011, 4: 3502-3508

Open Access This article is distributed under the terms of the Creative Commons Attribution License which permits any use, distribution, and reproduction in any medium, provided the original author(s) and source are credited. 